\title{
Mediterranean dietary pattern and non-alcoholic fatty liver diseases: a case-control study
}

\author{
Mohammad-Reza Entezari ${ }^{1}$, Nasir Talenezhad ${ }^{1}$, Farhang Mirzavandi ${ }^{1}$, Shahab Rahimpour ${ }^{1}$, \\ Hassan Mozaffari-Khosravi ${ }^{1}$, Hossein Fallahzadeh ${ }^{2}$ and Mahdieh Hosseinzadeh ${ }^{3,4} *$ (1) \\ ${ }^{1}$ Shahid Sadoughi University of Medical Sciences and Health Services, Yazd, Iran \\ ${ }^{2}$ Department of Biostatistics and Epidemiology, Research Center of Prevention and Epidemiology of Non-Communicable Disease, Faculty of Health, \\ Shabid Sadoughi University of Medical Sciences, Yazd, Iran \\ ${ }^{3}$ Nutrition and Food Security Research Center, Shabid Sadoughi University of Medical Sciences, Yazd, Iran \\ ${ }^{4}$ Department of Nutrition, School of Public Health, Shahid Sadoughi University of Medical Sciences, Yazd, Iran
}

(Received 9 June 2021 - Accepted 18 June 2021)

Journal of Nutritional Science (2021), vol. 10, e55, page 1 of 5

doi:10.1017/jns.2021.43

Abstract

The Mediterranean (MED) diet was associated with a reduced risk of chronic disease, but the epidemiological studies reported inconsistent findings related to the MED diet and non-alcoholic fatty liver disease (NAFLD) risk. This age and the gender-matched case-control study were conducted among 247 adult patients. The MED diet score was obtained based on the Trichopoulou model. Multivariate logistic regression was used to examine the association between the MED diet and NAFLD risk. NAFLD prevalence in people with low, moderate and high adherence to the MED diet was 33, 13.1 and $4 \cdot 6 \%$, respectively. The increasing intake of the MED diet was significantly related to the increment intake of nuts and fruits, vegetables, monounsaturated fatty acid/ polyunsaturated fatty acid ratio, legumes, cereals and fish. However, total energy consumption, low-fat dairy and meats intake were reduced $(P$ for all $<$ 0.05). Following control for age, the person in the highest of the MED diet tertile compared with the lowest, the odds of NAFLD decreased (OR: $0 \cdot 40,95$ $\%$ CI: $0 \cdot 17-0 \cdot 95)$. This relation became a little stronger after further adjusting for sex, diabetes, physical activity and supplement intake (OR: $0 \cdot 36,95 \%$ CI: $0 \cdot 15-0 \cdot 89)$. However, this association disappeared after adjusting for body mass index, waist and hip circumference (OR: 0.70, $95 \%$ CI: $0 \cdot 25-1 \cdot 97)$. High adherence to the MED diet was associated with a $64 \%$ reduction in NAFLD odds before some anthropometric variable adjustments. However, further prospective studies are required, particularly in BMI-stratified models.

Key words: Mediterranean diet: Non-alcoholic fatty liver disease: Dietary pattern

\section{Introduction}

Non-alcoholic fatty liver disease (NAFLD) is the most common cause of chronic liver disease worldwide ${ }^{(1)}$. NAFLD includes a wide range of liver damage, from simple steatosis to cirrhosis $^{(2)}$. NAFLD prevalence is estimated in adults worldwide, and it is evaluated to be about 20-25, 5-18 and $21 \cdot 5-31.5 \%$ in Asian countries and Iranian populations, respectively ${ }^{(3-5)}$. Obesity, diabetes, hyperlipidaemia, inadequate physical activity and an unhealthy diet are the most critical risk factors for NAFLD ${ }^{(6-9)}$.

Over the past decades, nutrition has been considered the dominant factor in preventing and treating non-communicable diseases, such as depression ${ }^{(10)}$ and NAFLD. Many studies demonstrated that deficiency in some micro- or macronutrients and high intake of some food groups could be related to $\mathrm{NAFLD}^{(10-12)}$. In recent years, epidemiological studies

Abbreviations: ALT: alanine aminotransferase; AST: aspartate aminotransferase; HC: hip circumference; MED: Mediterranean; MUFA/SAFA: monounsaturated fatty acid/ polyunsaturated fatty acid; NAFLD: non-alcoholic fatty liver diseases; TC: total cholesterol; TAG: triacylglycerols; WC: waist circumference

* Corresponding author: Mahdieh Hosseinzadeh, email hoseinzade.mahdie@gmail.com

(C) The Author(s), 2021. Published by Cambridge University Press on behalf of The Nutrition Society. This is an Open Access article, distributed under the terms of the Creative Commons Attribution licence (http://creativecommons.org/licenses/by/4.0/), which permits unrestricted re-use, distribution, and reproduction in any medium, provided the original work is properly cited. 
have shown that dietary patterns can better understand the correlation between dietary intake and NAFLD. The Mediterranean dietary score (MED diet) was proposed for the first time in $1995^{(13)}$. This dietary pattern was designed based on usual dietary intake and related to the risk of cardiovascular risk factors in MED countries. The MED diet includes a lot of beneficial fatty acids, fibre and micronutrients $^{(14)}$. Some epidemiological studies showed that NAFLD risk was reduced with increased adherence to the MED $\operatorname{diet}^{(15-20)}$, even though others could not find such a relationship $^{(15-17)}$. To the best of our knowledge, a few studies investigated the MED diet in developing countries, especially middle east countries where dietary patterns are different from other parts of the world. So, this case-control study aimed to examine the association between adherence to the MED diet and NAFLD in Iranian adults.

\section{Material and methods}

In this case-control study, we examined the 247 adult participants (108 men and 139 women) with an age range of 18-55 years old. NAFLD patients were diagnosed with abdominal ultrasonography and liver enzyme tests. Total requirement data were collected around medical history, anthropometry, physical activity and dietary intake. Afterwards, a trained dietitian and a face-to-face interview were used to gather a valid and reliable Food Frequency Questionnaire (FFQ). The participants reported the frequency and amount of consumption of each food item. Finally, all food items were converted to a gram per day and used for the final analysis.

The MED diet for the first time was expressed in 1995 and revised in 2003. In the revised and final model, Trichopoulou et al. considered nine components for the MED diet: vegetables, nuts and fruits, cereals, legumes, fish, monounsaturated fatty acid/polyunsaturated fatty acid ratio (MUFA/SAFA ratio), lowfat dairy, meats and alcohol intake. To calculate the MED diet score, all components were obtained based on a gram per day. These values were then converted as per $1000 \mathrm{kcal}$ energy intake, and sex-specific median values were used for the calculation scoring components based on the Trichopoulou method. Due to refusing to answer the questions related to alcohol consumption, this component was excluded, and finally, the MED diet score was calculated based on eight components.

We excluded patients with the following characteristics: overor under-reporting for energy intake ( $n$ 20), subjects providing incomplete information $(n 10)$ and subjects who had three or more components of metabolic syndrome ( $n$ 20). Finally, 197 subjects were involved in the analysis. Anthropometric data (height, weight and waist circumference (WC)) were measured by a trained person and a standard method. Fasting blood samples were collected after $12 \mathrm{~h}$ of fasting. The samples were immediately centrifuged and frozen at $-80^{\circ} \mathrm{C}$. Finally, at the end of sampling, the concentration of blood sugar, total cholesterol (TC), LDL, HDL, triacylglycerols (TAG), aspartate aminotransferase (AST) and alanine aminotransferase (ALT) were determined using a standard method.

The liver steatosis was estimated by evaluating the image brightness of the echo pattern. In this regard, patients with a total liver weight of lower than $33 \%$ were categorised as controls. Individuals were excluded at baseline in the case of (1) using drugs inducing hepatotoxicity (tamoxifen, steroids and amiodarone); (2) having cardiovascular diseases (coronary artery disease or congestive heart disease), diabetes type 1, chronic B or C hepatitis virus infections, cancer, Wilson's disease, haemochromatosis, biliary diseases or cirrhosis and another liver disease; and (3) having a history of being on a special diet. Laboratory data were collected from controls and NAFLD patients after more than $12 \mathrm{~h}$ of fasting in enrolment. To determine the serum concentrations of the hepatic enzymes as well as the glucose and lipid profiles, concentrations of liver enzymes, including ALT, AST and $\gamma$-glutamyltransferase, fasting blood glucose and lipid profiles, such as LDL-cholesterol, HDL-cholesterol, TC and TAG were determined.

After signing the informed consent forms, all participants underwent an abdominal ultrasound by the same radiologist using the same device. The liver steatosis was estimated by evaluating the image brightness of the echo pattern. Abdominal ultrasound cannot detect hepatic fat deposition in the case that it is less than $33 \%$ of the total liver weight. In this regard, patients with a total liver weight of lower than $33 \%$ were categorised as controls.

\section{Ethical consideration}

This study was conducted according to the guidelines laid down in the Declaration of Helsinki, and all procedures involving human subjects were approved by the Human Research Ethics Committees of Shahid Sadoughi University of Yazd Medical Sciences (No. IR.SSU.SPH.REC.1399.088). Written informed consent was obtained from all subjects after explaining the process of the present study.

\section{Statistical analysis}

SPSS software version 20 was used to perform all statistical analyses. Normality variables were examined with a histogram curve. To study continuous variables with normal and abnormal distribution, the one-way ANOVA and the KruskalWallis test were used, respectively. Multivariate logistic regression models were also used to assess the relationship between the MED diet score and NAFLD risk. The MED diet tertiles were composed, and the first tertile was considered as a reference group.

\section{Results}

In this study, the prevalence of NAFLD in people with low, moderate and high adherence to the MED diet was 33, 13.1 and $4.6 \%$, respectively. The means of age and the MED diet scores in the NAFLD group compared to the healthy group were $44.07 \pm 10 \cdot 36 v .43 \cdot 31 \pm 12 \cdot 15$ years and $4.05 \pm$ $1 \cdot 27 v \cdot 4 \cdot 16 \pm 1 \cdot 38$, respectively. The NAFLD group compared to the healthy group had higher BMI $(31.85 \pm 4.12 v$. $25.40 \pm 3.94, P<0.001)$, WC (102.50 $\pm 12.63 v .93 .30 \pm$ 9.78, $P<0 \cdot 001)$, TAG $(186.52 \pm 82.63 v .133 .07 \pm 59 \cdot 00, P$ 
Table 1. Demographic, anthropometric and biochemical characteristics of the study participants according to MDS tertiles

\begin{tabular}{|c|c|c|c|c|}
\hline & $\begin{array}{l}T 1 \\
\leq 4\end{array}$ & $\begin{array}{l}T 2 \\
\leq 5\end{array}$ & $\begin{array}{l}T 3 \\
\geq 6\end{array}$ & $P$-value ${ }^{\mathrm{a}}$ \\
\hline $\operatorname{MDS}(0-9)^{\mathrm{b}}$ & $3.26 \pm 0.83$ & $5.00 \pm 0.00$ & $6.24 \pm 0.51$ & 0.001 \\
\hline Age, year & $43.54 \pm 12 \cdot 00$ & $45 \cdot 57 \pm 10 \cdot 68$ & $41.41 \pm 10.91$ & 0.31 \\
\hline $\mathrm{BMI}, \mathrm{kg} / \mathrm{m}^{2}$ & $29 \cdot 35 \pm 7 \cdot 60$ & $28.47 \pm 5.51$ & $26 \cdot 13 \pm 3 \cdot 75$ & 0.07 \\
\hline WC, cm & $99 \cdot 24 \pm 13 \cdot 86$ & $97 \cdot 80 \pm 9.84$ & $93 \cdot 11 \pm 7 \cdot 45$ & 0.06 \\
\hline $\mathrm{HC}, \mathrm{cm}$ & $108 \cdot 02 \pm 10 \cdot 18$ & $106 \cdot 93 \pm 10 \cdot 92$ & $102 \cdot 30 \pm 6 \cdot 26$ & 0.02 \\
\hline FBS, mg/dl & $98(92-118)$ & $100(93-112)$ & $101(93-119)$ & 0.67 \\
\hline TAG, mg/dl & $163 \cdot 32 \pm 84 \cdot 23$ & $16 \cdot 20 \pm 67 \cdot 35$ & $146 \cdot 33 \pm 53 \cdot 90$ & 0.57 \\
\hline $\mathrm{TC}, \mathrm{mg} / \mathrm{dl}$ & $168 \cdot 77 \pm 41 \cdot 31$ & $174.95 \pm 42 \cdot 07$ & $163 \cdot 29 \pm 49 \cdot 52$ & 0.52 \\
\hline LDL, mg/dl & $105 \cdot 93 \pm 32 \cdot 61$ & $103 \cdot 73 \pm 31 \cdot 03$ & $113 \cdot 22 \pm 46 \cdot 35$ & 0.51 \\
\hline $\mathrm{HDL}, \mathrm{mg} / \mathrm{dl}$ & $42(38-47)$ & $42(35-48)$ & $43(36-50)$ & 0.98 \\
\hline AST, IU/I & $24.04 \pm 11.54$ & $23.25 \pm 12.43$ & $22 \cdot 62 \pm 12 \cdot 20$ & 0.84 \\
\hline ALT, IU/I & $29 \cdot 67 \pm 22 \cdot 38$ & $30 \cdot 52 \pm 19 \cdot 87$ & $29 \cdot 30 \pm 23 \cdot 40$ & 0.95 \\
\hline Male, $n(\%)$ & $52(26 \cdot 4)$ & $23(11.7)$ & $10(5 \cdot 1)$ & 0.35 \\
\hline Diabetes, $n(\%)$ & $35(17 \cdot 8)$ & $14(7 \cdot 1)$ & $7(3 \cdot 6)$ & 0.81 \\
\hline Physical activity, $n(\%)$ & $79(40 \cdot 1)$ & $27(13 \cdot 7)$ & $21(10 \cdot 72)$ & 0.55 \\
\hline Prevalence of NAFLD, $n(\%)$ & $65(33 \cdot 1)$ & $26(13 \cdot 2)$ & $9(4 \cdot 6)$ & 0.06 \\
\hline
\end{tabular}

MDS, Mediterranean dietary score; BMI, body mass index; WC, waist circumference; HC, hip circumference; FBS, fasting blood sugar; TAG, triacylglycerols; TC, total cholesterol; LDL, low-density lipoprotein; HDL, high-density lipoprotein; ALT, alanine aminotransferase; AST, aspartate aminotransferase.

${ }^{a}$ ANOVA or Kruskal-Wallis test for a continuous variable and $\chi^{2}$ test for a categorical variable.

${ }^{b}$ Per $1000 \mathrm{kcal}$ energy intake.

$<0 \cdot 001)$, TC $(177 \cdot 22 \pm 43 \cdot 73 v \cdot 160 \cdot 43 \pm 40 \cdot 86, P<0 \cdot 006)$, AST $(28 \cdot 60 \pm 14 \cdot 90 v \cdot 17 \cdot 60 \pm 5 \cdot 52, P<0 \cdot 001)$ and ALT $(39 \cdot 12 \pm 12.26 v \cdot 19 \cdot 76 \pm 8 \cdot 62, P<0.001)$ and lower HDL $(42 \cdot 08 \pm 9 \cdot 35$ v. 46.73 $\pm 18 \cdot 07, P<0 \cdot 023$ ) (data cannot be shown).

The participants in the top tertile of the MED diet score have the lower significant hip circumference (HC). There was no significant association between increasing the MED diet score tertiles and BMI, WC, FBS, lipid profile, AST and ALT (Table 1). A correlation between the MED diet score and liver enzymes was not observed in the Pearson test (AST $(r 0 \cdot 051, P 0 \cdot 48)$, ALT $(r 0 \cdot 042, P$ 0.056)).

The distribution of the MED diet components and macronutrient intake across the MED diet score tertiles are exhibited in Table 2. The increasing intake of the MED diet was significantly related to the increment intake of vegetables, nuts and fruits, cereals, legumes, fish and MUFA/SAFA ratio, albeit the consumption of total energy, low-fat dairy and meats intake was decreased. The relationship between the MED diet score and the risk of NAFLD is presented in Table 3. In the crude model, the individual in the highest MED diet tertile compared to the lowest tertile and the risk of NAFLD to $60 \%$ (OR: 0.40, 95\% CI: 0.17-0.95) were decreased. In model 1 , after controlling for sex, age, diabetes, physical activity and supplement intake, the relationship became stronger (OR: 0.36, $95 \% \mathrm{CI}$ : 0.15-0.89). However, in model 2, after adding BMI, WC and HC to model 1, this relationship disappeared (OR: 0.70, $95 \%$ CI: 0.25-1.97). Crude and adjusted OR for NAFLD according to MUFA/ SAFA ratio tertiles were reported in Table 4. Individuals in the highest MUFA/SAFA ratio tertiles have lower odds for NAFLD in crude and after adjusteing for age, sex, diabetes, physical activity and supplement intakead.

\section{Discussion}

The present study revealed a reverse association between high adherence to MED diet and NAFLD odds, even though this

Table 2. Distribution of the MED diet components and macronutrient intake across the MED diet score tertiles

\begin{tabular}{|c|c|c|c|c|}
\hline & $\begin{array}{c}T 1 \\
N 123\end{array}$ & $\begin{array}{c}T 2 \\
N 45\end{array}$ & $\begin{array}{c}T 3 \\
N 29\end{array}$ & $P$-value ${ }^{\mathrm{a}}$ \\
\hline Energy, kcal/d & $2352 \cdot 86 \pm 680 \cdot 80$ & $1924 \cdot 01 \pm 666 \cdot 40$ & $1702 \cdot 04 \pm 486 \cdot 72$ & 0.001 \\
\hline Vegetables, g/1000 kcal & $138 \cdot 04 \pm 83 \cdot 72$ & $165 \cdot 30 \pm 80 \cdot 06$ & $181 \cdot 76 \pm 65 \cdot 60$ & 0.001 \\
\hline Cereals, g/1000 kcal & $132 \cdot 87 \pm 55 \cdot 30$ & $139 \cdot 08 \pm 51 \cdot 73$ & $163 \cdot 15 \pm 60 \cdot 35$ & 0.031 \\
\hline Legumes, g/1000 kcal & $12 \cdot 86 \pm 8 \cdot 46$ & $18 \cdot 34 \pm 10 \cdot 85$ & $21 \cdot 30 \pm 9 \cdot 40$ & 0.001 \\
\hline Fruits and nuts, g/1000 kcal & $309 \cdot 39 \pm 155 \cdot 71$ & $362 \cdot 65 \pm 152 \cdot 14$ & $382 \cdot 42 \pm 148 \cdot 21$ & 0.013 \\
\hline Fish, g/1000 kcal & $5.93 \pm 10 \cdot 70$ & $5.79 \pm 3.48$ & $7 \cdot 21 \pm 7 \cdot 77$ & 0.021 \\
\hline Low-fat dairy, g/1000 kcal & $180(92-298)$ & $147(96-261)$ & $142(93-277)$ & 0.004 \\
\hline Meats, g/1000 kcal & $62 \cdot 80 \pm 31 \cdot 70$ & $54 \cdot 25 \pm 23 \cdot 70$ & $40 \cdot 54 \pm 18 \cdot 60$ & 0.001 \\
\hline MUFA/SAFA ratio, g/1000 kcal & $0.47 \pm 0.20$ & $0.59 \pm 0.24$ & $0.63 \pm 0.17$ & 0.001 \\
\hline Carbohydrate, $\mathrm{g} / \mathrm{d}$ & $329 \cdot 86 \pm 112 \cdot 36$ & $281 \cdot 87 \pm 114 \cdot 20$ & $262 \cdot 21 \pm 78 \cdot 10$ & 0.001 \\
\hline Protein, g/d & $100 \cdot 91 \pm 37.09$ & $80 \cdot 48 \pm 32 \cdot 24$ & $65 \cdot 38 \pm 22 \cdot 12$ & 0.001 \\
\hline Total fat, g/d & $82 \cdot 26 \pm 28 \cdot 39$ & $62.00 \pm 19.90$ & $52 \cdot 41 \pm 15 \cdot 34$ & 0.001 \\
\hline Tea and coffee, g/d & $624 \cdot 89 \pm 187 \cdot 17$ & $285 \cdot 84 \pm 215 \cdot 44$ & $240.52 \pm 192 \cdot 87$ & 0.001 \\
\hline
\end{tabular}

${ }^{\text {a }}$ ANOVA or Kruskal-Wallis test for a continuous variable and $\chi^{2}$ test for a categorical variable. 
Table 3. Crude and adjusted OR for NAFLD according to MED score tertiles

\begin{tabular}{|c|c|c|c|c|c|}
\hline MED & $\begin{array}{c}T 1 \\
\leq 4\end{array}$ & $\begin{array}{c}T 2 \\
5\end{array}$ & $\begin{array}{l}T 3 \\
\geq 6\end{array}$ & $P$ for trend & $P$-value \\
\hline Crude & 1.00 & $1.22(0.61-2.43)$ & $0.40(0.17-0.95)$ & 0.02 & 0.038 \\
\hline Model 1 & 1.00 & $1.20(0.60-2.47)$ & $0.36(0.15-0.89)$ & 0.04 & 0.027 \\
\hline Model 2 & 1.00 & $1.31(0.55-3.15)$ & $0.70(0.25-1.97)$ & $0 \cdot 13$ & 0.58 \\
\hline Model 3 & 1.00 & $1.60(0.63-3.05)$ & $0.88(0.30-2.65)$ & 0.22 & $0 \cdot 81$ \\
\hline Model 4 & 1.00 & $1.52(0.73-3.14)$ & $0.54(0.22-1.35)$ & 012 & 0.71 \\
\hline
\end{tabular}

Model 1: adjusted for age, sex, diabetes, physical activity and supplement intake.

Model 2: Model 1, additional BMI, WC and HC.

Model 3: Model 2, additional total energy intake.

Model 4: Adjusted for total energy intake.

relationship was disappeared after further adjustment for the anthropometric variable. Similar to some other studies, results, nested and matched case-control ${ }^{(16)}$ and cross-sectional ${ }^{(17)}$, reported that adherence to the MED diet in any crude and adjusted models was not associated with the risk of NAFLD. In addition, Kontogiani et al., in a cross-sectional study, did not observe a difference between adherence to the MED diet in NAFLD compared with control groups. However, this dietary pattern had an inverse association with the severity of NAFLD ${ }^{(18)}$. However, some studies inconsistent with the present study, including Bullon-Vela et al., claimed that greater adherence to the MED diet scores was inversely associated with hepatic steatosis index in a cross-sectional study of PREDIMED-PLUS, which is a large multi-centre clinical trial in the multivariate linear regression ${ }^{(19)}$. In a cross-sectional study, Barrata et al. reported that the MED diet score obtained based on a 9-item semi-quantitative questionnaire was negatively associated with the risk of NAFLD in the adjusted model ${ }^{(20)}$. Besides, Aller et al., in a cross-sectional study, indicated that higher adherence to the MED diet score was associated with a lower chance of a high grade of steatosis ${ }^{(21)}$. A recent metaanalysis also confirmed that Mediterranean dietary patterns reduced the risk of this disease by $23 \%{ }^{(22)}$.

The present study did not explore a significant relation between the MED diet score with ALT and AST. These results were similar to some other studies, including Della Corte et al., in which a relation between good, moderate and low adherence to KIDMED (the Mediterranean Diet Quality Index for children and adolescents) and the rise of ALT and AST were not observed ${ }^{(23)}$. Also, Tzima et al. illustrated that adherence to the MED diet in the metabolic syndrome patients was not relevant to reducing liver enzyme levels ${ }^{(24)}$.
The mean adherence to the MED diet score in the present study was $4 \cdot 02 \pm 1 \cdot 7$. It was similar to some other investigations ${ }^{(16)}$. It seems that the difference in the amount of sexspecific median intake of components in the MED diet could be related to the beneficial effects of this dietary pattern on NAFLD prevention. For example, males and females in the Trichopoulou study were compared to males and females in the present study who consumed more vegetables (median intake in male: 550 v. $278 \mathrm{~g} / \mathrm{d}$, in female: 500 v. $280 \mathrm{~g} / \mathrm{d}$ ), MUFA/SAFA ratio (median intake in male: $1.7 v .1$, in female: $1.7 v .0 .9$ ) and fish (median intake in male: $24 v .10 \mathrm{~g} / \mathrm{d}$, in female: $19 v .6 \mathrm{~g} / \mathrm{d})$.

Several mechanisms may be related to the beneficial effects of the MED diet on NAFLD because of the low content of simple sugars and fructose. The high content of soluble and insoluble fibres is associated with a decrease in serum TAG and blood glucose $^{(25)}$. This dietary pattern also has plenty of fruit, vegetables, olive oil and antioxidant compounds (twenty-eight), which may be particularly useful in NAFLD patients ${ }^{(26)}$.

The protective effect of the MED diet on the fatty liver was disappeared after further adjustment for BMI and WC and HC as central obesity, which shows that the MED Diet can play a role in improving fatty liver through weight modification, lipid profile and inflammation markers ${ }^{(27,28)}$. Several mechanisms have been proposed to explain the association between obesity and fatty liver, including changes in cytokines, inflammation factors, insulin resistance, dyslipidemia ${ }^{(29)}$ and regulation of the expression of some of the genes (PPAR- $\gamma$ and others) ${ }^{(30)}$. It is suggested to conduct future studies with more participants and BMI-matched case-control studies to determine the differences between groups more specifically.

Some strengths of the present study include the following issues: the questionnaires were completed by a trained

Table 4. Crude and adjusted OR for NAFLD according to MUFA/SAFA ratio tertiles

\begin{tabular}{lcccr}
\hline & $T 1$ & $T 2$ & $T 3$ & \\
MUFA/SAFA ratio & $\leq 0.42$ & $0.43-0.56$ & $\geq 0.57$ & $P$ for trend \\
\hline Crude & 1.00 & $1.33(0.66-2.67)$ & $0.47(0.24-0.95)$ & 0.013 \\
Model 1 & 1.00 & $1.30(0.63-2.62)$ & $0.42(0.20-0.87)$ & 0.03 \\
Model 2 & 1.00 & $1.85(0.78-4.35)$ & $0.71(0.28-1.76)$ & 0.04 \\
Model 3 & 1.00 & $2.50(0.88-7.11)$ & $1.32(0.30-6.10)$ & 0.14 \\
Model 4 & 1.00 & $1.90(0.81-4.44)$ & $0.92(0.30-2.92)$ & 0.11 \\
\hline
\end{tabular}

Model 1: Adjusted for age, sex, diabetes, physical activity and supplement intake.

Model 2: Model 1, additional BMI, WC and HC.

Model 3: Model 2, additional total energy intake.

Model 4: Adjusted for total energy intake. 
interviewer blinded to the participants' categorisation in the case or control group, which minimised the reporting error. Also, we used newly diagnosed individuals with NAFLD (incident case) as the case group. Various confounders associated with fatty liver and also dietary patterns were adjusted. The limitation of the present study includes the possibility of recall bias due to the nature of observation. However, in order to decrease this bias, a valid and reliable FFQ was used. Due to the case-control design of the study, determining a clear causal relationship was impossible between dietary pattern adherence and NAFLD. Since the case group members included newly diagnosed NAFLD patients, the probability of changing their dietary patterns was low after the disease diagnosis.

\section{Acknowledgments}

The authors thank all the patients for their participation and the Shahid Sadoughi University of Medical Sciences for supporting this research.

The authors declare that they have no competing interests.

The present study was supported by the Shahid Sadoughi University of Medical Sciences, Yazd, in Iran.

All the authors have contributed to drafting and revising the manuscript, study concept and design, as well as data gathering and interpretation. All the authors reviewed and approved the final version of the manuscript.

All authors declare no conflicts of interest.

\section{References}

1. Bellentani S, Scaglioni F, Marino M, et al. (2010) Epidemiology of non-alcoholic fatty liver disease. Dig Dis 28, 155-161.

2. Vuppalanchi R \& Chalasani N (2009) Nonalcoholic fatty liver disease and nonalcoholic steatohepatitis: selected practical issues in their evaluation and management. Hepatology 49, 306-317.

3. Williams CD, Stengel J, Asike MI, et al. (2011) Prevalence of nonalcoholic fatty liver disease and nonalcoholic steatohepatitis among a largely middle-aged population utilizing ultrasound and liver biopsy: a prospective study. Gastroenterology 140, 124-131.

4. Lankarani KB, Ghaffarpasand F, Mahmoodi M, et al. (2013) Non alcoholic fatty liver disease in southern Iran: a population based study. Hepat Mon 13, e9248.

5. Browning JD, Szczepaniak LS, Dobbins R, et al. (2004) Prevalence of hepatic steatosis in an urban population in the United States: impact of ethnicity. Hepatology 40, 1387-1395.

6. Trenell MI (2015) Sedentary behaviour, physical activity, and NAFLD: curse of the chair. J Hepatol 63, 1064-1065.

7. Ortiz-Lopez C, Lomonaco R, Orsak B, et al. (2012) Prevalence of prediabetes and diabetes and metabolic profile of patients with nonalcoholic fatty liver disease (NAFLD). Diabetes Care 35, 873-878.

8. Hamaguchi M, Kojima T, Takeda N, et al. (2005) The metabolic syndrome as a predictor of nonalcoholic fatty liver disease. Ann Intern Med 143, 722-728.

9. Tavakoli HR, Rahmati-Najarkolaei F, Malkami A, et al. (2018) The relation between fast food consumption and non-alcoholic fatty liver: a case-control study. Iran J Endocrinol Metab 20, 22-30.

10 Maryam K, Gity S, Maryam A, et al. (2020) The relationship between dietary patterns and depression mediated by serum levels of Folate and vitamin B12. BMC Psychiatry 20(1), http://dx.doi.org/10.1186/ s12888-020-2455-2.
11. Ferolla SM, Ferrari TCA, Lima MLP, et al. (2013) Dietary patterns in Brazilian patients with nonalcoholic fatty liver disease: a crosssectional study. Clinics 68, 11-17.

12. Machado RM, Stefano JT, Oliveira CP, et al. (2010) Intake of trans fatty acids causes nonalcoholic steatohepatitis and reduces adipose tissue fat content. J Nutr 140, 1127-1132.

13. Trichopoulou A, Kouris-Blazos A, Wahlqvist ML, et al. (1995) Diet and overall survival in elderly people. Br Med J 311, 1457-1460.

14. Tosti V, Bertozzi B \& Fontana L (2018) Health benefits of the Mediterranean diet: metabolic and molecular mechanisms. J Gerontol A Biol Sci Med Sci 73, 318-326.

15. Yang C-Q, Shu L, Wang S, et al. (2015) Dietary patterns modulate the risk of non-alcoholic fatty liver disease in Chinese adults. Nutrients 7, 4778-4791.

16. Park S-Y, Noureddin M, Boushey C, et al. (2020) Diet quality association with nonalcoholic fatty liver disease by cirrhosis status: the multiethnic cohort. Curr Dev Nutr 4, nzaa024.

17. Chan R, Wong VW-S, Chu WC-W, et al. (2015) Diet-quality scores and prevalence of nonalcoholic fatty liver disease: a population study using proton-magnetic resonance spectroscopy. PLoS One 10, e 0139310

18. Kontogianni MD, Tileli N, Margariti A, et al. (2014) Adherence to the Mediterranean diet is associated with the severity of nonalcoholic fatty liver disease. Clin Nutr 33, 678-683.

19. Bullon-Vela V, Abete I, Tur JA, et al. (2019) Influence of lifestyle factors and staple foods from the Mediterranean diet on nonalcoholic fatty liver disease among older individuals with metabolic syndrome features. Nutrition 71, 110620.

20. Baratta F, Pastori D, Polimeni L, et al. (2017) Adherence to Mediterranean diet and non-alcoholic fatty liver disease: effect on insulin resistance. Am J Gastroenterol 112, 1832-1839.

21. Aller R, Izaola O, de la Fuente B, et al. (2015) Mediterranean diet is associated with liver histology in patients with non alcoholic fatty liver disease. Nutr Hosp 32, 2518-2524.

22. Zadeh SH, Mansoori A \& Hosseinzadeh M (2020) Relationship between dietary patterns and non-alcoholic fatty liver disease: A systematic review and meta-analysis. J Gastroenterol Hepatol 36, 1470-1478.

23. Della Corte C, Mosca A, Vania A, et al. (2017) Good adherence to the Mediterranean diet reduces the risk for NASH and diabetes in pediatric patients with obesity: the results of an Italian study. Nutrition 39-40, 8-14.

24. Tzima N, Pitsavos C, Panagiotakos DB, et al. (2009) Adherence to the Mediterranean diet moderates the association of aminotransferases with the prevalence of the metabolic syndrome: the ATTICA study. Nutr Metab 6, 30.

25. Garcia M, Bihuniak JD, Shook J, et al. (2016) The effect of the traditional Mediterranean-style diet on metabolic risk factors: a meta-analysis. Nutrients 8, 168.

26. Spahis S, Delvin E, Borys J-M, et al. (2017) Oxidative stress as a critical factor in nonalcoholic fatty liver disease pathogenesis. Antioxid Redox Signal 26, 519-541.

27. Richard C, Couture P, Desroches S, et al. (2013) Effect of the Mediterranean diet with and without weight loss on markers of inflammation in men with metabolic syndrome. Obesity 21, 51-57.

28. Esposito K, Kastorini C-M, Panagiotakos DB, et al. (2011) Mediterranean diet and weight loss: meta-analysis of randomized controlled trials. Metab Syndr Relat Disord 9, 1-12.

29. Jung UJ \& Choi M-S (2014) Obesity and its metabolic complications: the role of adipokines and the relationship between obesity, inflammation, insulin resistance, dyslipidemia and nonalcoholic fatty liver disease. Int J Mol Sci 15, 6184-6223.

30. Pettinelli P \& Videla LA (2011) Up-regulation of PPAR- $\gamma$ mRNA expression in the liver of obese patients: an additional reinforcing lipogenic mechanism to SREBP-1c induction. J Clin Endocrinol Metab 96, 1424-1430. 